\title{
Caracterización de Líquidos por Técnica Fotoacústica: Estudio de Sensores Piezoeléctricos de Banda Ancha
}

\author{
L. Cusato*, M. Estevez*, G. Santiago*1 and M. G. González*†2 \\ *Universidad de Buenos Aires, Facultad de Ingeniería, \\ Grupo de Láser, Óptica de Materiales y Aplicaciones Electromagnéticas (GLOMAE) \\ Paseo Colón 850, C1063ACV, Buenos Aires, Argentina \\ ${ }^{1}$ gsantiaefi.uba.ar \\ ${ }^{\dagger}$ Consejo Nacional de Investigaciones Científicas y Técnicas, (CONICET) \\ C1425FQB, Buenos Aires, Argentina \\ ${ }^{2}$ mggonza@fi.uba.ar \\ Recibido: 22/05/17; Aceptado: 03/07/17
}

\begin{abstract}
In this paper we present the study of three sensors, based on piezoelectric polymer thin films, for photoacoustic systems used in the characterization of liquids. Its performance was compared by carrying out measurements on well-known liquids. From the measured data, the sensitivity, noise level and quantification limit of each of the sensors were determined. The device with the best characteristics was used in the determination of the quantum efficiency of two dyes: Rhodamine 6G and Rhodamine $B$. The measured values agree very well with those reported by other authors.
\end{abstract}

Resumen - En este trabajo se presenta el estudio sobre tres sensores para sistemas fotoacústicos usados en caracterización de líquidos basados en películas delgadas de polímero piezoléctrico. Su desempeño fue comparado llevando a cabo mediciones sobre líquidos de características bien conocidas. A partir de los datos medidos se determinó la sensibilidad, el nivel de ruido y el límite de cuantificación de cada uno de los sensores. Aquel con las mejores características fue utilizado en la determinación de la eficiencia cuántica de los colorantes Rodamina 6G y Rodamina B. Los valores medidos concuerdan muy bien con los reportados por otros autores.

\section{INTRODUCCIÓN}

La técnica fototérmica (FT) es valorada en áreas como medición de trazas de gases, determinación de propiedades de líquidos y sólidos o formación de imágenes virtuales de objetos opacos. Entre otras virtudes se cuentan la simpleza, el relativo bajo costo y la flexibilidad para aplicarlas a numerosos procesos de medida [1].

La técnica FT está basada en la absorción de radiación electromagnética por parte de la muestra bajo estudio. Cuando las moléculas absorben la radiación, se genera un estado excitado que, al relajarse en forma no radiativa, produce un calentamiento en la muestra. Este calentamiento genera ondas de presión y cambios en las propiedades ópticas que pueden ser medidos a través de diferentes detectores. Si la magnitud medida son las ondas de presión, la técnica usualmente se denomina fotoacústica (FA) [2].

Todo esquema experimental FA consta de tres partes esenciales [1]: i) una fuente de excitación, usualmente un láser; ii) el conjunto recinto-sensor, donde se coloca la muestra y se mide la señal FA generada; iii) y el procesado de la señal captada para obtener de ella la información.

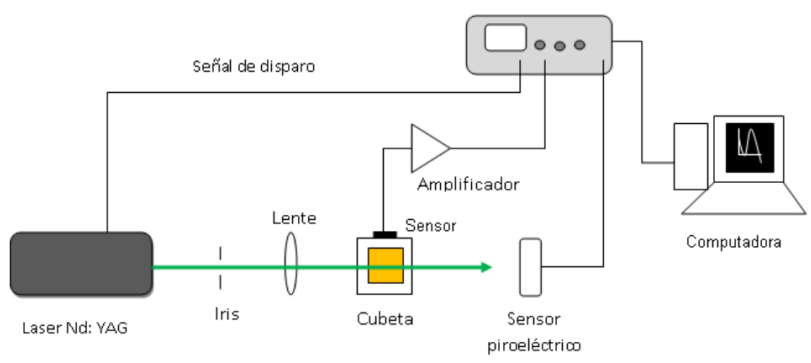

Fig. 1. Configuración experimental típica para medición de líquidos usando la técnica FA

Los requerimientos generales buscados en un sistema FA son: alta sensibilidad, selectividad, amplio rango dinámico, mediciones en tiempo real, portabilidad y bajo costo. Para lograrlos, deben estudiarse y desarrollarse mejoras sobre cada uno de los componentes esenciales.

En este trabajo se presenta el estudio sobre tres conjuntos recinto-sensor de sistemas FA para la determinación de la eficiencia cuántica de fluorescencia de líquidos.

\section{LA TÉCNICA FA EN LÍQUIDOS}

Un sistema FA típico para caracterización de líquidos [3] se muestra en la Fig. 1 donde se pueden apreciar las tres partes esenciales detalladas en la sección anterior. Como fuente de excitación se tiene un láser pulsado cuya energía es medida por un detector piroeléctrico. El haz es limitado espacialmente por un iris y su ancho es adecuado a través del uso de una lente convergente. El conjunto recinto sensor se encuentra conformado por una cubeta y un sensor acústico adosado en una de sus paredes. La señal captada por el sensor es amplificada y posteriormente digitalizada por un osciloscopio. Finalmente los datos son procesados en una computadora para obtener los parámetros buscados.

La energía transmitida a la muestra, caracterizada por el coeficiente de absorción óptico $\mu$ y el camino recorrido en la muestra $l$, está dada por [4]:

$$
E_{t}=E_{0} e^{-\mu l}
$$


con $E_{0}$ la energía del láser incidente. La energía absorbida por la muestra es:

$$
E_{a}=E_{0}\left(1-e^{-\mu l}\right)
$$

Además, el coeficiente de absorción se puede calcular como $\mu=C \epsilon$, con $C$ la concentración de la muestra y $\epsilon$ el coeficiente de extinción de la misma.

Si los efectos radiativos y las reacciones químicas fotoinducidas pueden ser desechados, entonces toda la energía absorbida se convierte en calor. Este calor determina la generación de pulsos acústicos debidos a una expansión adiabática. La amplitud de esta presión depende linealmente con la energía absorbida por la muestra [4]:

$$
p \propto \frac{\beta c^{2}}{C_{p} l} E_{a}=\frac{\beta c^{2}}{C_{p} l} E_{0}\left(1-e^{-\mu l}\right)
$$

donde $\beta$ es el coeficiente de expansión térmica, $c$ la velocidad del sonido y $C_{p}$ el calor específico.

Así como en los espectrómetros UV-visible, en estos sistemas FA es usual el uso de una cubeta para contener la muestra a ser analizada. Para este tipo de recinto, las ondas de presión generadas por un haz láser colimado (el ancho espacial no varía con la distancia) podrán ser esféricas, cilíndricas o una combinación de ambas dependiendo del valor de $\mu$, que a su vez define una longitud de penetración $\delta$. Si $\delta>>l$, se generan ondas cilíndricas cuyo eje es la región de la muestra que absorbió energía láser. Si $\delta<<l$, se tiene una fuente acústica puntual lo que llevará a la generación de ondas esféricas. En la situación intermedia, $\delta \simeq l$, se generarán ondas de los dos tipos [4]. La forma de la onda de presión también depende del perfil espacial del haz láser que incide sobre la muestra. En este trabajo las mediciones siempre fueron hechas para $\delta>>l$. Para esta condición y si el haz láser es enfocado, la fuente de ondas acústicas es una pequeña región alrededor del foco, aproximable a una fuente puntual. Por lo tanto, la forma de onda de presión dependerá principalmente del perfil espacial del haz láser dentro de la cubeta.

En el caso en que $\mu l<<1$, la presión generada es lineal con la absorbancia del material $A=\mu l=\epsilon C l$ :

$$
p \propto \frac{\beta c^{2}}{C_{p} l} E_{0} \mu l
$$

\section{A. Dependencia temporal de la fuente láser}

Como se desprende de lo descripto más arriba, es esencial conocer la dependencia temporal del haz láser. En todas las experiencias llevadas a cabo en este trabajo se utilizó un láser Nd:YAG Q-switched pulsado doblado en frecuencia (Continuum Minilite I, $532 \mathrm{~nm}, 10 \mathrm{~Hz}$, polarización vertical).

Para determinar el perfil temporal del haz se utilizó un fotodiodo (Motorola MRD500, 1ns, -18 V). Un polarizador de Nicoll fue usado para atenuar la energía del láser captada por el fotodiodo. De esta manera se protege la integridad del fotodiodo y se evitan saturaciones que degraden el ancho de banda del detector y la forma temporal que se desea medir [5]. La tensión de salida entregada por el fotodiodo fue digitalizada por un osciloscopio (Tektronix TDS2024B, 2 $\mathrm{GS} / \mathrm{s}, 200 \mathrm{MHz}$ ) y procesada en una computadora personal.

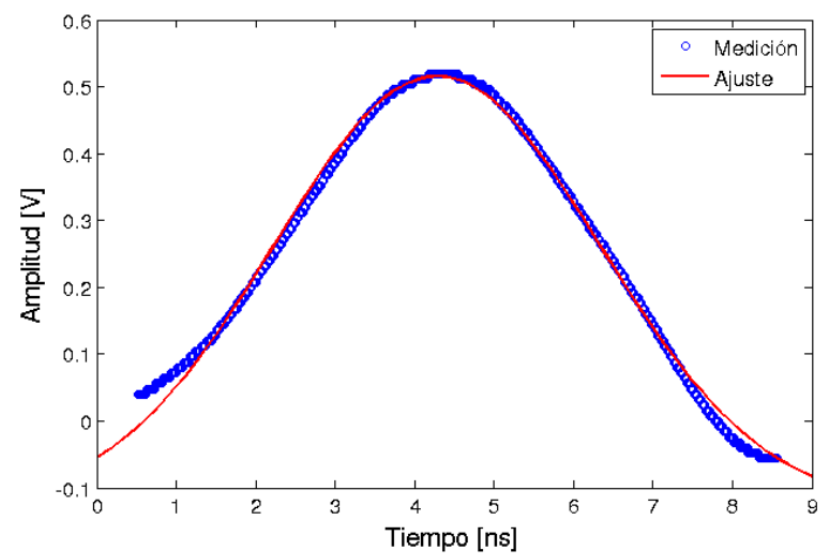

Fig. 2. Señal fotodiodo medida (asteriscos) y ajuste con función gaussiana (línea llena).

La señal para sincronizar el disparo del osciloscopio fue obtenida del pulso de Q-switch. La señal medida se muestra en la Fig. 2. La medición fue ajustada a un perfil gaussiano obteniéndose un ancho a mitad de altura de $4.8 \mathrm{~ns} \pm 2$ ns.

\section{B. Medición de la energía de un pulso láser}

La amplitud de la presión acústica, a una dada absorbancia, depende de la energía láser incidente. Por lo tanto para la caracterización de un líquido es necesario conocer su valor. En este trabajo se utilizó un detector piroeléctrico (Coherent LMP10) para verificar la estabilidad del pulso láser en el tiempo. Para ello se midió la energía cada 1 minuto durante 30 minutos. Para digitalizar la señal entregada por el detector se usó el mismo osciloscopio de la subsección anterior.

Se encontró que la energía del haz varía como máximo un $3 \%$, y que esta diferencia se debe más a la discretización del osciloscopio que a una variación real del pulso.

En muchos casos fue necesario llevar a cabo las experiencias con energías del orden o inferiores a la mínima energía detectable por el dispositivo piroeléctrico $(0.7 \mathrm{~mJ})$; lo que conlleva a mediciones de energía muy ruidosas. Para solucionar esto, se decidió filtrar el ruido a través del uso de redes neuronales [6]. La red escogida para el filtrado pertenece al tipo perceptron multicapa. El esquema implementado presenta 1000 neuronas en la capa de entrada, 2 capas ocultas con 20 neuronas cada una y una capa de salida con 1000 neuronas. Los patrones de entrenamiento de la red se generaron a partir de una señal medida con una buena relación señal a ruido (SNR). Es importante destacar que mientras mayor es la variabilidad y cantidad de patrones, mayor será la capacidad para recuperar la señal inmersa en ruido. Sin embargo, esto está limitado al procesador y memoria de la computadora utilizada. Generalmente, se escoge un error de entrenamiento teniendo en cuenta todos los parámetros antes mencionados. En la Fig. 3 se puede apreciar el funcionamiento de la red como filtro de señales piroeléctricas corrompidas por ruido incoherente.

\section{Sensores Piezoeléctricos De Banda AnCha}

En este trabajo se presenta el estudio sobre tres sensores basados en polímero piezoeléctrico. Los polímeros piezoeléctricos, como el polifluoruro de vinilideno (PVDF) y sus copolímeros, son muy usados en la construcción de 


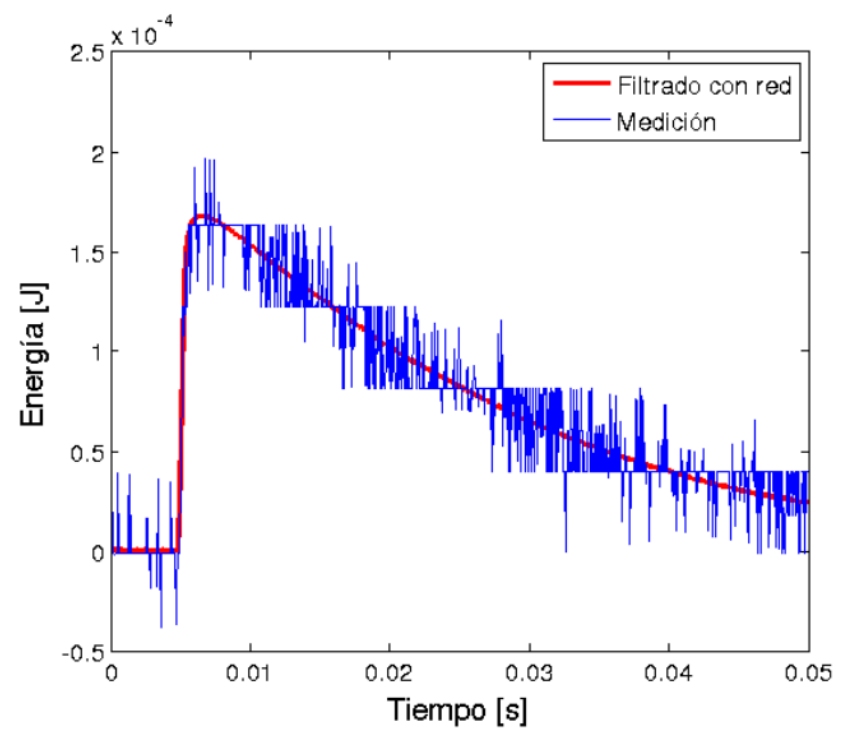

Fig. 3. Señal piroeléctrica a energías bajas $(<0.7 \mathrm{~mJ})$. Línea azul: medida. Línea roja: filtrada.

sensores debido a sus propiedades físicas que permiten su utilización en gran cantidad de aplicaciones. Estos materiales son flexibles, están disponibles como películas delgadas, tienen un gran ancho de banda acústica, y sus valores de impedancia acústica están próximos al del agua lo que los vuelve interesantes para su aplicación en el estudio de líquidos [7], [8]. Las propiedades aquí detalladas dependen, entre otras cosas, fuertemente de cómo el film es adherido al substrato o cuerpo del sensor. Se probaron tres esquemas cuyo desempeño fue comparado llevando a cabo mediciones sobre dos líquidos de características bien conocidas (Anilina y Orange II). A partir de las mediciones se determinó la respuesta temporal, la sensibilidad, el límite de cuantificación y el nivel de ruido de cada uno de los sensores.

Los tres sensores fueron implementados con películas delgadas piezoeléctricas mono-orientadas de PVDF comercial (Piezotech SA, Saint Louis, Francia) de $25 \mu \mathrm{m}$ de espesor.

El sensor I consiste en una cubeta para espectroscopía $\left(4 \mathrm{~cm}^{3}\right)$ que posee adherido a una de sus paredes mediante pintura de plata (Electroquímica Delta SRL, Argentina) el PVDF con forma circular (6 mm de diámetro) y encapsulado en un conector BNC estándar. Este recinto-sensor fue usado en un esquema similar al detallado en la Fig. 1, con una lente de distancia focal de $50 \mathrm{~mm}$.

Para el sensor II se pegaron dos cubetas como las del sensor I, y se colocó en medio un film cuadrado de $9 \mathrm{~mm}$ de lado. El esquema experimental utilizado se muestra en la Fig. 4. Lo que se buscó en este caso fue lograr un aumento de la amplitud medida en un factor dos a través de la mayor compresión del film conseguida por las ondas de presión generadas en las cubetas. Como se requerían dos haces que incidieran en ambas cubetas a la vez, se utilizó un separador de haz de cuarzo para dividir el haz incidente.

El dispositivo III es similar al sensor I pero la cubeta tiene un volumen menor $\left(1.6 \mathrm{~cm}^{3}\right)$ y el film de iguales dimensiones se encuentra adherido con epoxy conductivo (Circuitworks Planned products, Santa Cruz CA). Este sensor se utilizó en el mismo esquema experimental que para

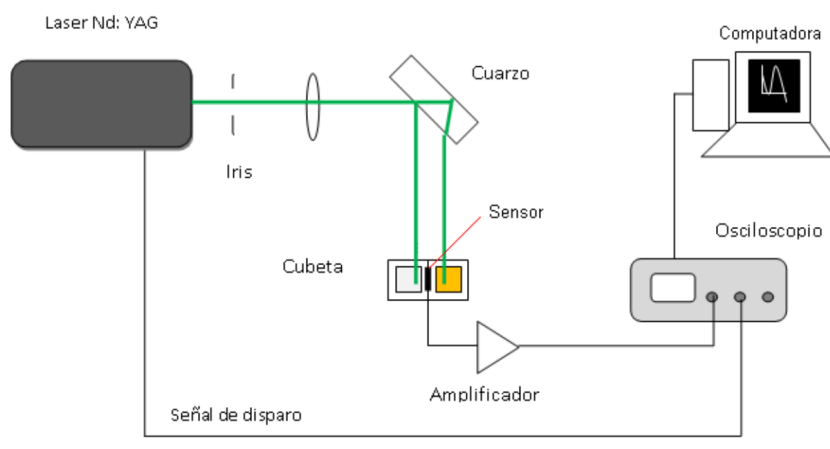

Fig. 4. Configuracón experimental para el sensor II.

el caso del transductor I.

El potencial gran ancho de banda de la película delgada de PVDF sólo puede aprovecharse cuando la resistencia de carga del mismo es lo suficientemente reducida como para dar una constante de tiempo igual o inferior al período de la señal a ser medida. Este sensor se comporta, primariamente, como una fuente de corriente en paralelo con un capacitor de decenas de picofaradios [9]. Este equivalente eléctrico muestra que es imposible conectar un sensor de este tipo a la entrada de un amplificador de tensión (como la de un osciloscopio), porque el ancho de banda efectivo resultaría de apenas unas pocas decenas de $\mathrm{kHz}$. Tampoco serviría reducir la resistencia de carga dado que la responsividad del sistema sería demasiado pequeña. Por estos motivos, es necesario utilizar un amplificador de transimpedancia con baja impedancia de entrada y gran ancho de banda. En el estudio llevado a cabo aquí se usó el amplificador marca Femto modelo HCA-100M-50K-C (50 kV/A, 100 MHz@ 10 $\mathrm{pF})$.

\section{A. Análisis de la respuesta temporal}

Se midió la respuesta temporal de los tres sensores para los colorantes mencionados anteriormente. Una señal típica medida por el sensor I del colorante anilina disuelto en agua deionizada se muestra en la Fig. 5. Cómo se describió en la sección II, la dependencia temporal del pulso láser es aproximadamente de tipo gaussiano. Considerando que la relajación del medio es rápida, es lógico suponer una onda de presión con la misma dependencia temporal. A su vez, la carga generada en los electrodos del film piezoeléctrico es proporcional a esta presión. Sin embargo, lo que es amplificado es la corriente, con lo cual la señal medida es proporcional a la derivada del pulso. Se puede apreciar que la onda principal generada en el centro de la cubeta aparece a $\operatorname{los} 3.40 \mu \mathrm{s}$, con respecto al pulso láser. Este retardo se relaciona con el hecho de que el pulso de presión atraviesa $5 \mathrm{~mm}$ de líquido, y luego $1 \mathrm{~mm}$ de cuarzo (pared de la cubeta). Por otro lado, en los primeros $100 \mathrm{~ns}$ de la señal medida se pudo apreciar un ruido coherente proveniente del láser (Fig. 5a), producto de un blindaje electromagnético imperfecto por parte del sensor [9]. Además, se observaron una serie de réplicas de la onda principal a los $3.9 \mu \mathrm{s}$ (Fig. 5c), que son debidas a rebotes de la onda principal dentro de la pared de la cubeta que contiene al sensor. La respuesta temporal no depende del colorante utilizado.

Para los otros sensores se realizó un análisis similar al 


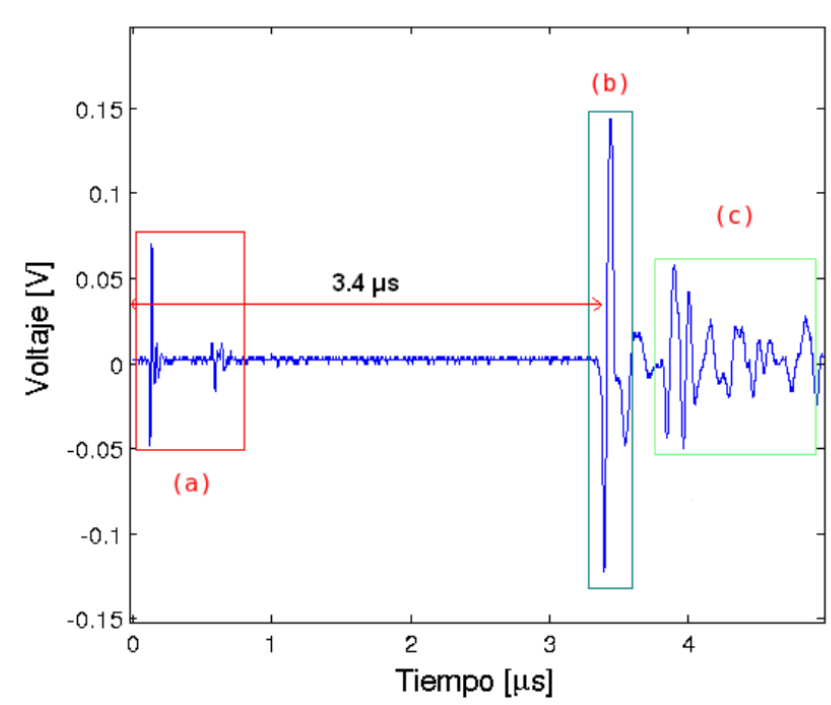

Fig. 5. Señal medida por sensor I de una muestra de anilina en agua. a) señal electromagnética generada por el Q-switched b) señal de presión c) rebotes de la señal de presión en la pared de la cubeta

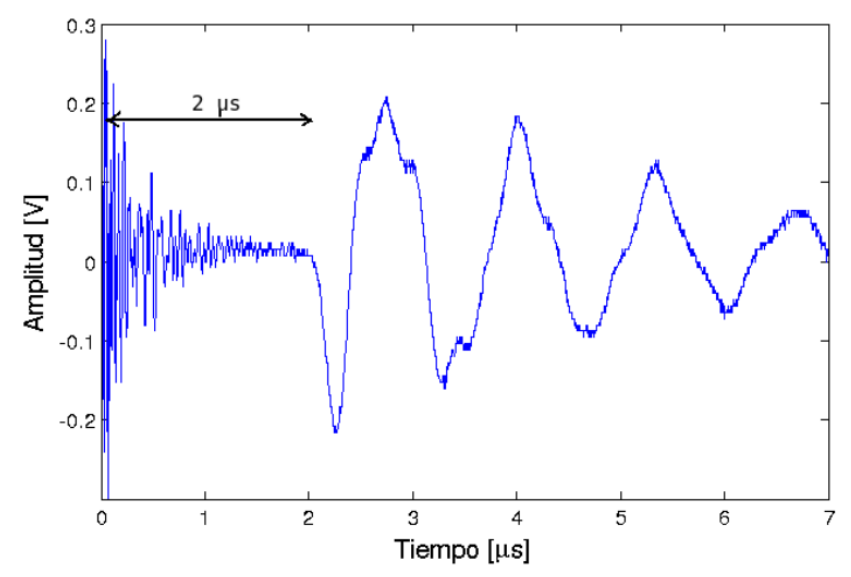

Fig. 6. Señal sensor II para Orange II en etanol.

anterior. Las señales típicas para el colorante Orange II en etanol se muestran en las Figs. 6 y 7. Los tiempos medidos para estos sensores difireren de los anteriores acorde a que que las distancias entre la fuente acústica y el sensor y los espesores de los recintos que contienen la muestra son diferentes.

En el caso particular del sensor II, se realizó una experiencia a fin de constatar el funcionamiento esperado del dispositivo (obtener una duplicación de la señal). Para ello se midió la señal obtenida para una solución de Orange II. Primero se irradió una cubeta y se obtuvo la respuesta, luego se repitió para la otra y, finalmente, se hizo la suma de la mediciones. Por otro lado, se irradió ambas cubeta al mismo tiempo. Se comprobó que la medición de todo el sistema en simultáneo era la suma de las señales de cada cubeta. La dificultad de este sistema reside en la correcta alineación del mismo, ya que para lograr una duplicación de la amplitud es necesario que ambas señales provenientes de las cubetas, lleguen al sensor al mismo tiempo. Luego de un largo proceso de alineación se logró el objetivo buscado y el resultado se muestra en la Fig. 8.

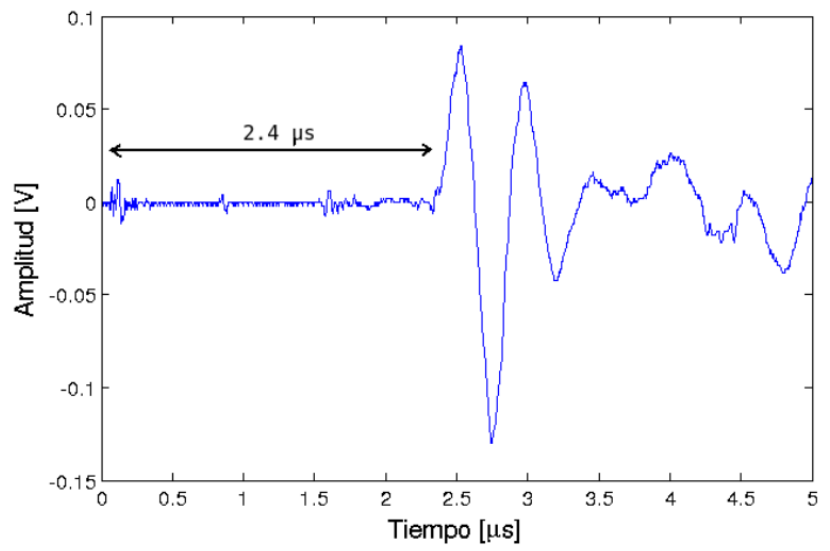

Fig. 7. Señal sensor III para Orange II en etanol.

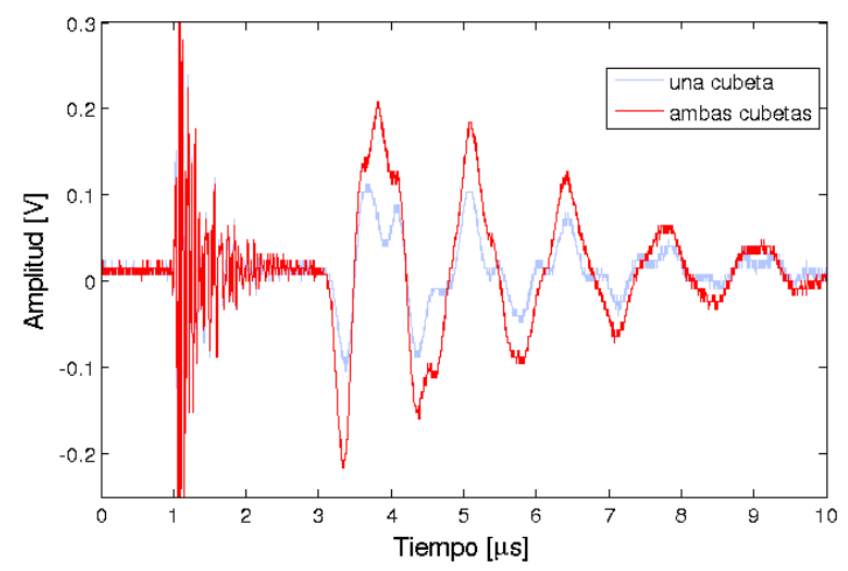

Fig. 8. Señal sensor III cuando el láser incide en una cubeta (gris) y cuando ingresa en las dos cubetas en simultáneo (rojo).

\section{B. Sensibilidad y señal de fondo}

Los tres sistemas estudiados fueron calibrados usando distintas muestras de Orange II (Merck, $99.9 \%$ ) en etanol absoluto (Acidic EtOH, Dorwil, etanol 99.5\%, $\mathrm{H}_{2} \mathrm{O}$ máximo $0,2 \%$ ). En la Fig. 9 se muestran los espectros medidos para distintas diluciones. Se utilizó un espectrómetro Shimadzu UV-1800 para medir la absorbancia de las muestras.

En la Fig. 10 se muestra los resultados correspondientes al sensor I con su ajuste en la zona lineal. Cómo estimador se utilizó la amplitud pico a pico de las señales, y se las normalizó con los valores de energías correspondientes a las mediciones. Además, se midió la amplitud de la señal de fondo (etanol puro), que corresponde al límite de cuantificación del sistema. Gráficos similares al mostrado en la Fig. 10 se obtuvieron para los sensores II y III.

\section{Nivel de Ruido}

Para medir el nivel de ruido presente en un señal se midió la amplitud pico a pico del mismo y se tuvo en cuenta el ancho de banda espectral del sistema. Como se detalló en la sección II el ancho de banda del conjunto sensor-amplificador depende principalmente de la capacidad eléctrica equivalente del sensor. De esta manera, se procedió a calcular el nivel de ruido como $V_{p p} / \sqrt{A B}$ donde $A B$ es el ancho de banda del sistema sensor-amplificador. Siguiendo 


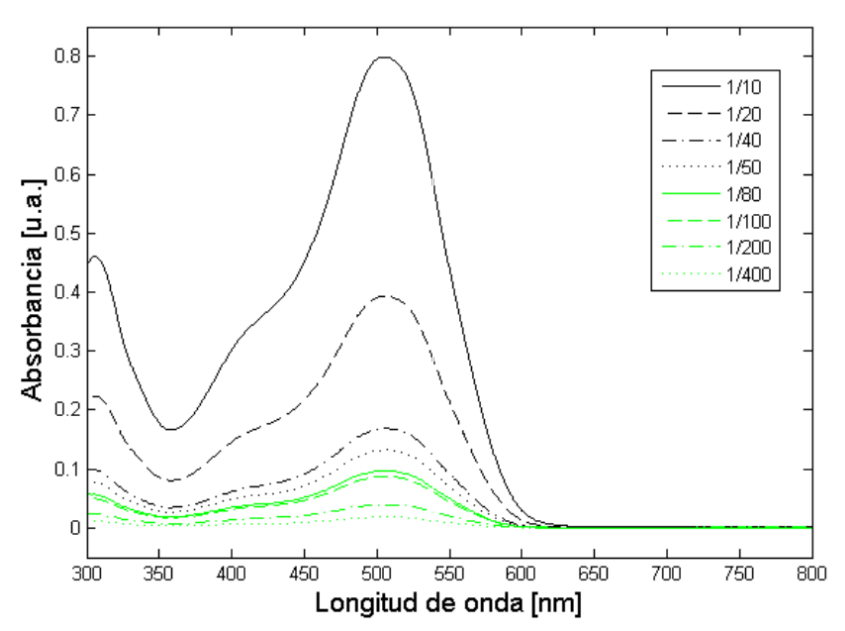

Fig. 9. Espectros de las distintas diluciones de Orange II utilizadas.

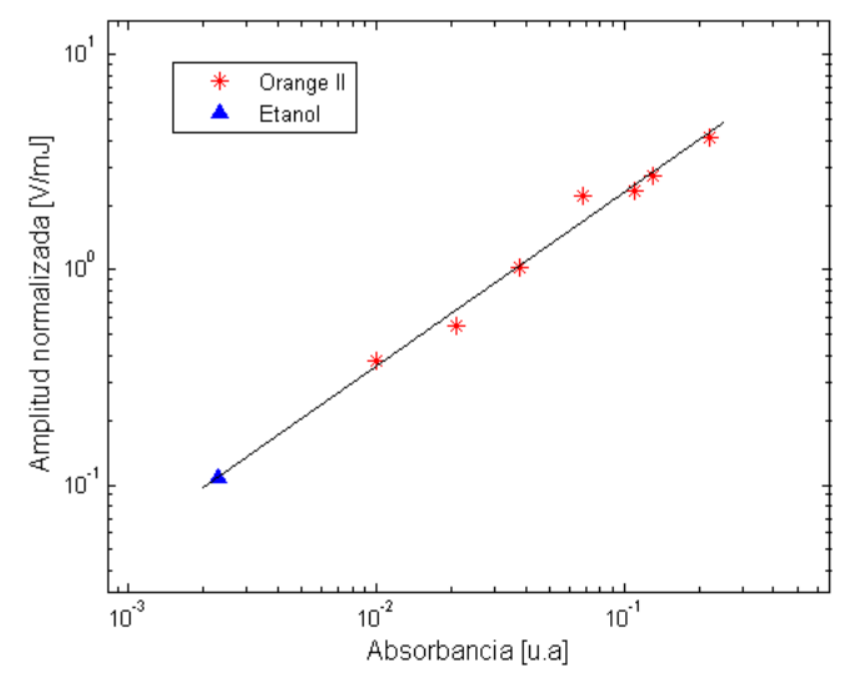

Fig. 10. Amplitud normalizada de las señales FA en función de la absorbancia. Asterisco: Orange II + etanol. Línea sólida: ajuste lineal. Triángulo: límite de cuantificación del sistema (medición etanol puro)

la metodología reportada en [9], para los sensores I y III se determinó un $A B=40 \mathrm{MHz}$, mientras que para el sensor II $A B=10 \mathrm{MHz}$, debido a que posee una capacidad eléctrica equivalente mayor.

\section{Comparación de los sensores}

En la Fig. 11 se muestra un cuadro donde se comparan las características de los distintos sensores estudiados.

El sensor II es el más sensible pero con el peor valor de

\begin{tabular}{|c|c|c|c|}
\hline \multirow{2}{*}{ Característica } & \multicolumn{3}{|c|}{ Sensor } \\
\cline { 2 - 4 } & I & II & III \\
\hline Sensibilidad $[\mathrm{V} / \mathrm{mJ}]^{a}$ & 14.72 & 21.35 & 1.89 \\
\hline Nivel de ruido $[\mathrm{mV} / \sqrt{\mathrm{MHz}}]$ & 1.075 & 2.656 & 1.138 \\
\hline Límite de cuantificación & $2,29.10^{-3}$ & $2,20.10^{-3}$ & $2,33.10^{-3}$ \\
\hline Cantidad de muestra & $4 \mathrm{ml}$ & $8 \mathrm{ml}$ & $1.6 \mathrm{ml}$ \\
\hline Complejidad del esquema experimental & sencilla & compleja & sencilla \\
\hline
\end{tabular}

Fig. 11. Cuadro comparativo de los sensores estuadiados. ${ }^{a}$ La sensibilidad se define como la amplitud normalizada por la energía del haz láser y la absorbancia, que se corresponde con la pendiente de la recta de calibración. El límite de cuantificación del sensor es la absorbancia de la mínima señal de presión medible, que corresponde a la señal de fondo (etanol puro). límite de detección dado que tiene el mayor nivel de ruido. Además, presenta la dificultad de alineación mencionada más arriba. El sensor I posee una sensibilidad similar al sensor II con un nivel de ruido menor y un sencillo esquema experimental. Finalmente, el sensor III también presenta bajo nivel ruido y requiere un pequeño volumen de muestra y posee una menor sensibilidad que los otros sensores.

Un análsis posterior mostró que la mayor sensibilidad del sensor I es debida a la mayor adherencia del PVDF al substrato (en este caso vidrio). Esto sugiere que la pintura de plata sería una mejor opción que el epoxy para actuar como adhesivo conductivo.

Para la determinación de la eficiencia cuántica de sustancias luminiscentes, objetivo de la siguiente sección, era necesario emplear poca solución por cada medición, por lo que se recurrió al sensor III, pese a ser el de menor sensibilidad.

\section{Determinación de la EFiciencia CuÁntica}

Para cualquier tipo de sustancia fotoluminiscente, la eficiencia cuántica de su luminiscencia $\phi_{f}$ representa una de sus propiedades más importantes. El conocimiento de $\phi_{f}$ provee información de los procesos no radiativos en moléculas y, en el caso de colorantes orgánicos, es esencial para seleccionar un medio láser eficiente. Además su medición es importante para distintas aplicaciones biomédicas [10], [11].

La eficiencia cuántica expresa una medida directa de la conversión de fotones absorbidos $N_{a b s}$ en fotones emitidos $N_{e m}$ :

$$
\phi_{f}=\frac{N_{e m}}{N_{a b s}}
$$

Para su cálculo es necesario considerar los procesos radiativos y no radiativos que se dan en el medio analizado. En el caso en que los procesos radiativos de relajación pueden ser despreciados, entonces la energía absorbida se convierte sólo en calor [4].

Los métodos convencionales de medición requieren de la comparación de la muestra bajo estudio con otra de $\phi_{f}$ conocida [12], y la exactitud de la medición se encuentra limitada por la confiabilidad de la técnica de comparación aplicada.

Para soluciones transparentes, el método más elemental para calcular $\phi_{f}$ consiste en comparar la intensidad de fluorescencia de una solución de un fluoróforo con la de otra de $\phi_{f}$ conocida, bajo las mismas condiciones de medición. En caso se usan espectrómetros de fluorescencia y absorción convencionales. Algunos inconvenientes que presenta este método surgen del valor de referencia usado. El uso de una esfera integradora es una posible solución para que la $\phi_{f}$ de referencia utilizada presente la menor incerteza posible. La misma permite medir $N_{a b s}$ y $N_{e m}$ directamente. La desventaja es que presenta una calibración compleja y la necesidad de corregir los efectos de reabsorción.

Por otro lado, la $\phi_{f}$ puede ser calculada indirectamente mediante la medición de la fracción de radiación incidente convertida en calor. En este caso se usan métodos fototérmicos como, por ejemplo, la espectroscopía FA detallada en la sección II. La técnica FA tiene como ventaja 
una relativamente simple calibración con la muestra no fluorescente de referencia y, en general, posee una gran sensibilidad. Además, los métodos fototérmicos miden con gran exactitud y son altamente reproducibles.

La amplitud de la onda de presión $p$ generada en la muestra al ser irradiada, ec. 4, puede reescribirse teniendo en cuenta la relajación radiativa [12]:

$$
p\left(\lambda_{e x}\right)=k \varsigma \frac{\beta c^{2}}{C_{p} l} E_{0} A\left(\lambda_{e x}\right)=k \varsigma \alpha\left(\lambda_{e x}\right)
$$

donde $k$ es un factor de corrección relacionado con la muestra y la medición, $\lambda_{e x}$ la longitud de onda de excitación y $\varsigma$ la relación entre la relajación radiativa y no radiativa. Cuando los procesos de relajación radiativos (por ejemplo la fluorescencia) o las reacciones químicas fotoinducidas pueden ser descartados, $\varsigma$ es igual a 1. Para una muestra fluorescente con especies que emiten y absorben sólo una vez, la ec. 6 puede ser reescrita como [12]:

$$
p\left(\lambda_{e x}\right)=\alpha\left(\lambda_{e x}\right) k\left(1-\frac{\lambda_{e x}}{\lambda_{e m}} \phi_{f}\right)
$$

donde $\lambda_{e m}$ es la longitud de onda de emisión máxima del espectro del fluorósforo, $\alpha$ es directamente proporcional a la fracción de luz absorbida por la muestra y el término entre paréntesis representa la pérdida por fotoactividad de la señal FA. Como se mencionó anteriormente, la eficiencia del fluorósforo se mide relativa a una sustancia de referencia que no fluoresce (conversión enteramente a calor) en las mismas condiciones de medición. Para esta sustancia, donde no hay procesos radiativos,

$$
p_{\text {ref }}=\alpha k
$$

Luego, se puede obtener la razón entre $p$ y $p_{\text {ref }}$ a partir del término de pérdida radiativa:

$$
\frac{p\left(\lambda_{e x}\right)}{p_{r e f}\left(\lambda_{e x}\right)}=1-\frac{\lambda_{e x}}{\lambda_{e m}} \phi_{f}
$$

Conociendo $\lambda_{e m}$ puede obtenerse $\phi_{f}$, despejando de la ecuación anterior:

$$
\phi_{f}=\frac{\lambda_{e x}}{\lambda_{e m}}\left(1-\frac{p\left(\lambda_{e x}\right)}{p_{r e f}\left(\lambda_{e x}\right)}\right)
$$

\section{A. Colorantes luminiscentes y de referencia}

Se probó el sistema con dos compuestos de la familia de las rodaminas. Las rodaminas son derivados xanténicos que se utilizan en la implementación de láseres. Sus espectros de emisión se encuentran entre los $500 \mathrm{~nm}$ y $700 \mathrm{~nm}$ [13] y poseen rendimientos cuánticos de fluorescencia elevados [14]. Las rodaminas son solubles tanto en agua como en alcoholes, y sus propiedades espectroscópicas se ven influenciadas por la interacción con estos solventes [15]. En este trabajo se utilizaron soluciones de Rodamina 6G (R6G, Exciton Inc, Rhodamine 590 Chloride, dye content $>99 \%$ ) y Rodamina B (RB, Exciton Inc, Rhodamine 610, dye content $>99 \%$ ) disueltas en etanol absoluto (Acidic EtOH, Dorwil, etanol 99,5\%, $\mathrm{H}_{2} \mathrm{O}$ máximo 0,2 \%).

La Rodamina 6G es utilizada comúnmente como medio amplificador para láseres de colorante, por este motivo es de interés el estudio de su $\phi_{f}$. En soluciones acuosas con

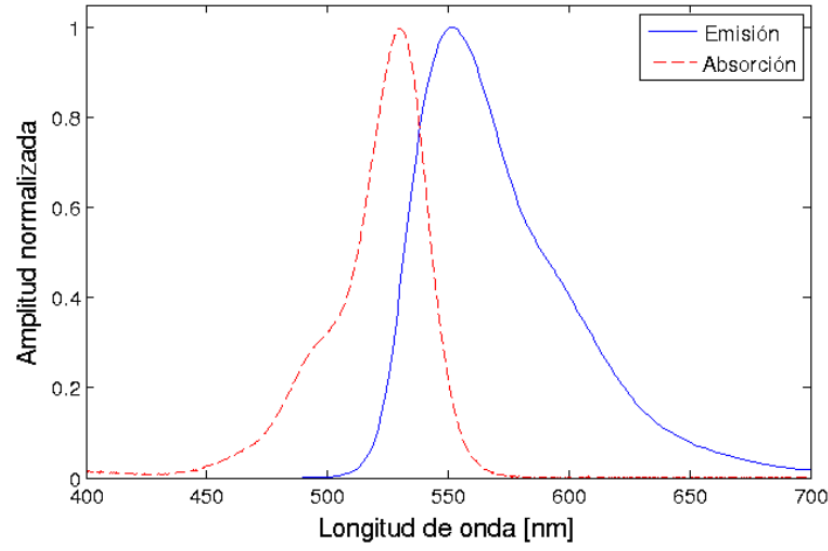

Fig. 12. Espectros de absorción (línea punteada) y de emisión (línea llena) de la Rodamina 6G

concentraciones cercanas a $10^{-5}$ M la concentración de dímeros no puede despreciarse en la determinación de $\phi_{f}$. En cambio, en soluciones alcohólicas como el etanol, la tendencia a formar dímeros se ve reducida y éstos no se observan hasta concentraciones del orden de $0.1 \mathrm{M}$ [11]. Basado en esto, para las concentraciones usadas en este trabajo, puede despreciarse el efecto de éstos en los cálculos. Esta rodamina presenta su máximo de absorción en $530 \mathrm{~nm}$ y su máximo de emisión en $564 \mathrm{~nm}$, este valor puede variar según la concentración. En la Fig. 12 se observa el espectro de absorción y de emisión para una concentración del orden de las utilizadas aquí (datos obtenidos de www.exciton.com). La longitud de onda del láser utilizada en éste trabajo se encuentra muy cercano al pico de absorción de la R6G, por lo cual se espera que una proporción grande de la energía incidente sea absorbida.

La Rodamina B presenta su máximo de absorción en $564 \mathrm{~nm}$ y su máximo de emisión en $580 \mathrm{~nm}$ cuando es disuelta en etanol. En la Fig. 13 se detallan los espectro de emisión y absorción de la Rodamina B (datos obtenidos de www.exciton.com). Dado que el máximo del espectro de emisión de la RB se encuentra más alejado de la longitud de onda de excitación, se espera hallar una absorción menor que para la R6G, con un consecuente menor valor de $\phi_{f}$.

Como sustancia de referencia para la medición de $\phi_{f}$ se utilizaron los colorantes Fuchsin (Sigma Aldrich, Basic Fuchsin, dye content $>88 \%$ ) y Orange II (Merck, 99,9 \%) ya que ninguna de ellas presenta luminiscencia. El Fuchsin es utilizado para aplicaciones biológicas como la coloración de bacterias, como desinfectante y además es ampliamente usado como referencia para medición de $\phi_{f}$. Tiene su pico de absorción en $544 \mathrm{~nm}$. El Orange II, también conocido como acid orange 7 , es otro colorante muy utilizado en biología. Tiene su máximo de absorción en $483 \mathrm{~nm}$. En la Fig. 14 se muestran los espectros de absorción medidos para los colorantes de referencia utilizados.

\section{B. Degradación de los colorantes}

Se realizaron mediciones a fin de verificar que no hubiese formación de dímeros en las soluciones de rodamina, los cuales afectarían al valor de $\phi_{f}$ calculado. Con este objetivo se utilizó un dispositivo experimental similar al descrito en la Fig. 1 pero, en lugar del sensor, se colocó una cubeta de 


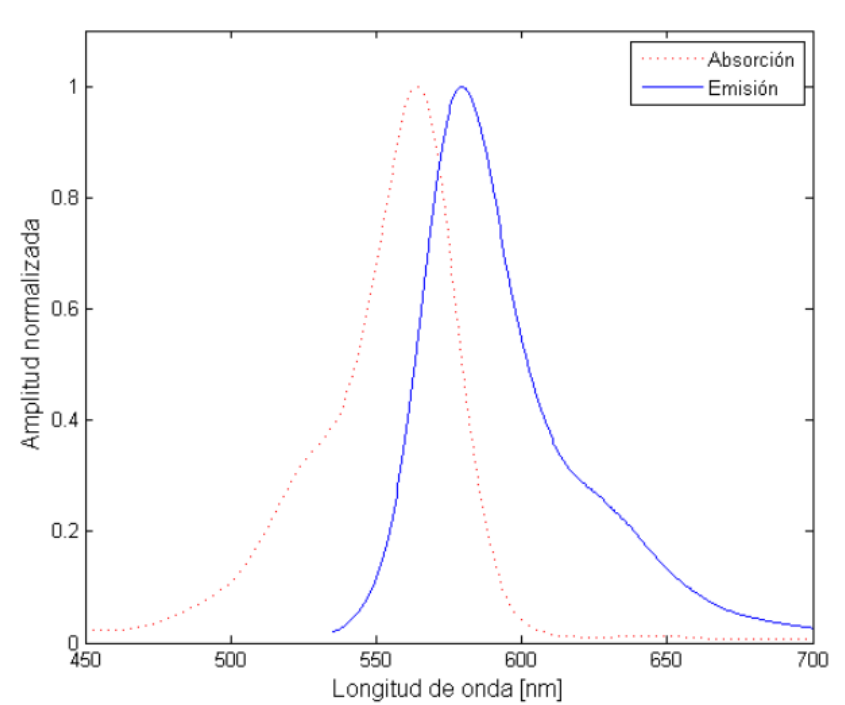

Fig. 13. Espectros de absorción (línea punteada) y de emisión (línea llena) de la Rodamina B

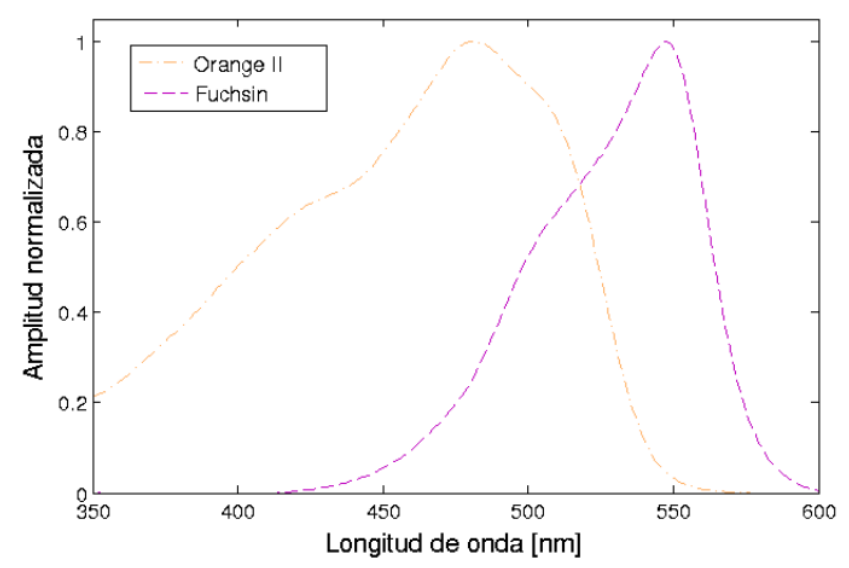

Fig. 14. Espectros de absorción de Orange II (naranja) y Fuchsin (violeta)

espectroscopía con una solución de rodamina en etanol. Se prepararon 6 muestras idénticas; 5 fueron irradiadas con el láser para distintas energías del mismo. Luego se midió la absorbancia de cada una de ellas. A mayor energía, es más factible la producción de dímeros.

Para el colorante R6G se usó una solución con concentración de $10^{-5} \mathrm{M}$, que según lo mencionado en la subsección anterior se encuentra dentro del rango donde no se observa formación de dímeros por alta concentración. Los resultados arrojaron que la variación entre las absorbancias fue menor a $1,7 \%$. Se realizó el mismo experimento para el colorante RB obteniéndose un valor de $6 \%$. Dado que las absorbancias medidas oscilan alrededor de un valor medio, consideramos que este diferencia no se debe a la degradación del colorante por irradiación láser sino a las fluctuaciones del instrumento de medición, en este caso el espectrofotómetro.

\section{Rectas de calibración y cálculo de $\phi_{f}$}

Se realizaron las rectas de calibración para todos los colorantes, siguiendo el proceso descrito en la sección III. Con el objetivo de estimar el error, el procedimiento fue realizado repetidas veces. Es importante remarcar que, una

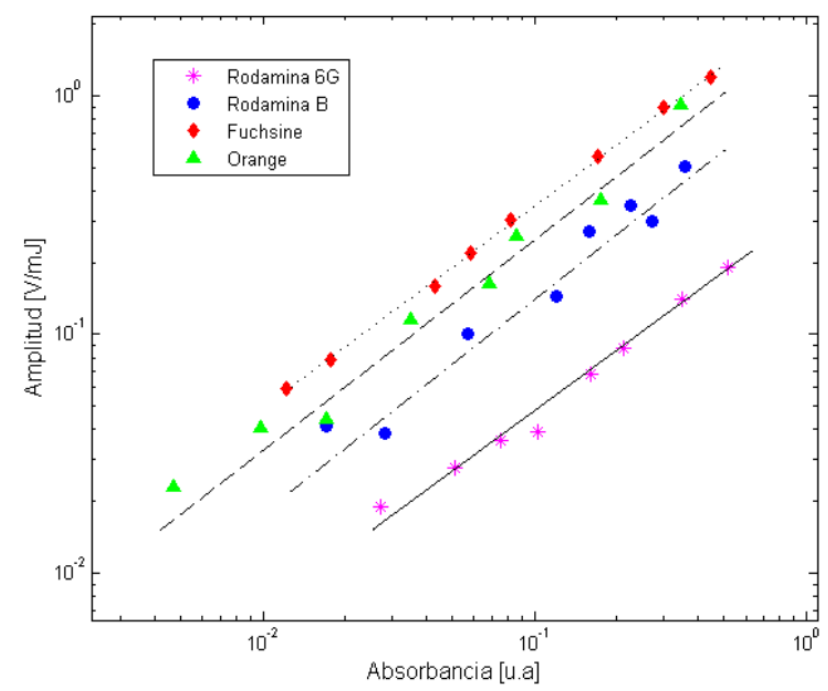

Fig. 15. Mediciones de señales para Fuchsin (rombos), Orange II (triángulos), Rodamina B (cículos) y Rodamina 6G (asteriscos). Las líneas son el resultado de los ajustes lineales de los datos.

\begin{tabular}{|c|c|c|}
\hline Colorante luminiscente & Referencia & Eficiencia cuántica \\
\hline \hline \multirow{2}{*}{ Rodamina 6G } & Orange & $0,88 \pm 0,07$ \\
\cline { 2 - 3 } & Fuchsin & $0,92 \pm 0,02$ \\
\hline \multirow{2}{*}{ Rodamina B } & Orange & $0,46 \pm 0,37$ \\
\cline { 2 - 3 } & Fuchsin & $0,59 \pm 0,23$ \\
\hline
\end{tabular}

Fig. 16. Efciencias cuánticas obtenidas para los colorantes luminiscentes con distintas referencias.

vez alineado el sensor, debía mantenerse inmóvil durante todas las mediciones. Por lo tanto no podía retirarse la cubeta para llenarla con la dilución correspondiente. Por esta razón, se utilizó una jeringa para extraer el contenido de la cubeta, y el llenado se hizo volcando directamente la solución; cuidando de no mojar el sensor. Al analizar todas las mediciones correspondientes a un mismo colorante, se lavó repetidas veces la cubeta con agua de alta pureza para evitar que quedaran restos de solución que interfirieran en futuras mediciones. Finalmente, se graficó la amplitud normalizada de las señales de la onda de presión en función de la absorbancia. Teniendo en cuenta la ec. 8, se realizó un ajuste lineal de los datos, que establece la relación entre la presión y la absorbancia (ver ec. 6) para cada referencia. En la Fig. 15 se muestran las mediciones para los colorantes de referencia y los colorantes luminiscentes, junto con sus ajustes lineales.

A partir de la ec. 10 y las pendientes de las curvas de calibración fue posible hallar los valores de $\phi_{f}$ correspondiente a los colorantes luminiscentes utilizados. Los valores obtenidos se detallan en el cuadro comparativo de la Fig. 16. Los mismos se encuentran dentro del rango obtenido por otros autores: 0.87-0.96 para Rodamina 6G en etanol [16], [17] y 0.49-0.65 para Rodamina B en etanol [17], [18].

\section{CONCLUSIÓN}

Se montaron y caracterizaron tres diseños de sensores FA. Para cada sensor se determinó la sensibilidad, el nivel de ruido y el límite de cuantificación. Se encontró que el sensor II es el más sensible. Sin embargo, el nivel de ruido 
y la dificultad a la hora de alinear el sistema lo vuelven poco adecuado. Para el caso de los sensores I y III, el sistema FA es mucho más sencillo. La sensiblidad del sensor I fue mayor que la del sensor III porque la pintura de plata resultó mejor adhesivo que el epoxy. Sin embargo, se eligió utilizar el sensor III para las mediciones de los colorantes luminiscentes dado que posee un volumen reducido.

En la determinación de $\phi_{f}$ se utilizaron dos colorantes de referencia distintos (Orange II y Fuchsin) con propiedades similares, y dos colorantes luminiscentes (Rodamina B y 6G). Los valores encontrados de $\phi_{f}$ para ambas referencias concuerdan muy bien con los reportados por otros autores.

\section{AGRADECIMIENTOS}

Los autores agradecen a la Universidad de Buenos Aires $\mathrm{y}$ al CONICET por la financiación de este trabajo a través de los subsidios UBACyT 20020120100025BA y 20020130100346BA, y PIP-112-201101-00676.

\section{REFERENCIAS}

[1] M. G. González, "Análisis y Desarrollo de Nuevas Técnicas de Detección Optoacústicas", Tesis de Doctorado, Universidad de Buenos Aires, 2008.

[2] C. Haisch, "Photoacoustic spectroscopy for analytical measurements", Meas. Sci. Technol. vol. 23, p. 012001, 2012

[3] A. Rosencwaig, "Photoacoustics and photacoustic sprectroscopy", John Wiley and Sons Inc., Nueva York, 1981.

[4] T. Schmid, U. Panne, R. Niessner, C. Haisch, "Optical absorbance measurements of opaque liquids by pulsed láser photoacoustic spectroscopy”, Anal. Chem. vol. 81, pp. 2403-2409, 2009.

[5] H.G. Arrigo, "Optimización de sistemas de detección de señales ópticas para alta potencia y alta velocidad", Tesis de grado de Ingeniería Electrónica, Facultad de Ingeniería de la Universidad de Buenos Aires, 2011.

[6] M. González, A. Peuriot, V. Slezak, G. Santiago, "Recovery of noisy pyroelectric-detector signals through neural-network processing”, Rev. Sci. Intrum. vol. 76, p. 053104, 2005

[7] L. F. Brown, ?Design considerations for piezoelectric polymer ultrasound transducers,? Ultrasonics, Ferroelectrics, and Frequency Control, IEEE Transactions on, vol. 47, no. 6, pp. 1377?1396, 2000.

[8] M. G. González, P. A. Sorichetti and G. Santiago, "Modeling thinfilm piezoelectric polymer ultrasonic sensors", Rev. Sci. Instrum. vol 85, no. 11, 2014, doi: 10.1063/1.4901966.

[9] M. González, P. Sorichetti, G. Santiago, "Caracterización de sensores planos de polímero piezoeléctrico para tomografía optoacústica", Argencon 2014 IEEE Conference Publications, pp. 281-285, 2014

[10] J.E.Selwyn, J.I.Steinfeld, "Aggregation equilibria of xanthene dyes", J.Phys.Chem. vol. 76, pp. 762-774, 1972

[11] K.Igarashi, M.Maeda, T.Takao, Y.Oki, H. Kusama, ”Dominant Factors of Preventing Rhodamine 6G from Dimer Formation in Aqueous Solutions", Bull Chem Soc Japan vol. 72, pp. 1197-1202, 1999

[12] J.N. Demas, G.A.Crosby, "The Measurement of Photoluminescence Quantum Yields", Phys. Chem. vol. 75, pp. 991-1024, 1971

[13] B. Soffer, B. McFarland, "Continously tunable, narrow-band organic dye lasers", Appl. Phys.Lett. vol. 10, pp. 266-267, 1967.

[14] B.Valeur, "Molecular Fluorescence Principles and Applications", Wiley-VCH Verlag GmbH, 1ra edición, Weinheim, 2001.

[15] G. Saini, A. Sharma, S. Kaur, K. Bindra, V. Sathe, S. Tripathi, C. Mhahajan, "Rhodamine $6 \mathrm{G}$ interaction with solvents studied by vibrational spectroscopy and density functional theory", J. Mol. Struct. vol. 931, pp. 10-19, 2009.

[16] L. Porrès, A. Holland, L. Palsson, A. Monkman, C. Kemp, A. Beeby, "Absolute measurements of photoluminiscence quantum yields of solutions using an integrating sphere", Journal of Fluorescence vol. 16, pp. 267-273, 2006.

[17] R. Kubin, A Fletcher, "Fluorescence quantum yields of some rhodamine dyes", J. Luminescence, vol. 27, pp. 455-462, 1982.

[18] K. Casey, E. Quitevis, "Effect of solvent polarity on nonradiative processes in xanthene dyes: Rhodamine B in normal alcohols", J. Phys. Chem. vol. 92, pp. 6590-6594, 1988. 